\title{
Linear and Non-Linear Optical Characterization of MnS Nanoparticle
}

\author{
Shabna .S \\ Department of Physics, Sree Narayana College Chengannur
}

\begin{abstract}
Non linear optics is given increasing attention due to its wide application in the area of laser technology, optical communication and data storage technology. Intense research has been fueled by the need for practical optical device that can deal the deficiencies of conventional technologies. The leading materials could have very high bulk second order NLO values well beyond those available today, which substantially reduces the cost of fabrication of electro-optic devices. The present review focus on the linear and non-linear optical properties of MnS nanoparticle and its future scope.
\end{abstract}

\section{Introduction}

The nanoparticles and nanostructured materials have attracted great interest because of their properties such as quantum confinement of electrons and holes, surface effects, and geometrical confinement of phonons are different from those of bulky materials. Nanoparticles have a rather large number of atoms, but its size is comparable with characteristic dimensions describing the behavior of electrons and holes, thus creating an intermediate regime between molecules and bulk crystals.

The optical responses of metal nanoparticles, nanoapertures in metal films and Meta materials are focuses on enhancing local electromagnetic fields to enormous facilitate light matter interactions. The enormous enhancement factors of $10^{3}-10^{6}$ compared to the fundamental electric field at a flat metal surface has been predicted and these strong local fields are particularly important for non-linear optical processes, such as second harmonic generation and third harmonic generation.

\section{The Z-Scan Technique}

The Z-Scan technique is a simple and most sensitive experimental technique to measure intensity dependent nonlinear susceptibilities of materials. The technique is particularly useful when the non-linear refraction is accompanied by non-linear absorption.

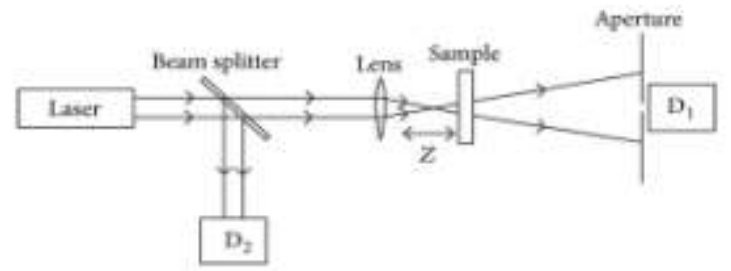

Figure 1: The Z-scan experimental setup in which the ratio $\mathrm{D}_{2} / \mathrm{D}_{1}$ is recorded as a function of the sample position $\mathrm{z}$

In Z-Scan experiments, the transmittance of a sample is measured through a finite aperture in the far field as the sample is moved along the propagation path of a Gaussian beam. It is possible to separately evaluate the non-linear refraction as well as the non-linear absorption by performing a second Z-Scan with the aperture removed.
The interaction of the medium with the laser light changes as the sample is moved. By measuring the transmittance through the sample as a function of the z-position of the sample, information about the light matter interaction can be extracted.

When a sample is far away from the focus, nonlinear refraction is negligible and the transmittance remains relatively constant. As the sample moves closer to the focus, the beam irradiance increases, leading to self-lensing in the sample. A maximum transmittance through the aperture will occur when the sample is just in front of the focus. The maximum in transmittance will drop to minimum as the sample is moved further and the beam diverges as a result of the negative lensing by the sample. Thus a material with negative nonlinearity gives a prefocal peak followed by a post focal valley as a Z-scan signature. Similarly a material with positive nonlinearity, gives rise to an opposite valleypeak configuration.

\subsection{Reagents and Apparatus}

Dilute $\mathrm{HNO}_{3}$, deionized distilled water, EDTA, $\mathrm{MnCl}_{2}$, $\mathrm{Na}_{2} \mathrm{~S}$, Acetone, two $50 \mathrm{ml}$ beakers, two $100 \mathrm{ml}$ beakers, measuring jar, magnetic stirrer, glass rods, glass rods, digital weighing machine, centrifuging machine etc.

\subsection{Preparation OF MnS Nanoparticle (CO - Precipitation Method)}

MnS nanoparticles of present study are prepared through simple chemical precipitation method using Manganese chloride $\left(\mathrm{MnCl}_{2}\right)$ as precursor. The principle involved in this technique is the precipitation of metal ions with Chloride ions in the solution. 0.1 M aqueous solution of Manganese Chloride and 0.1 M aqueous solution of Sodium Sulphide were mixed in the presence of E.D.T.A as capping agent. First solutions of $50 \mathrm{ml} \mathrm{MnCl} 2$ and $50 \mathrm{ml} \mathrm{Na}_{2} \mathrm{~S}$ were prepared in double distilled water. $10 \mathrm{ml}$ of $\mathrm{MnCl}_{2}$ and $5 \mathrm{ml}$ of E.D.T.A were mixed together and stirred for 30 minutes on a magnetic stirrer to get a homogeneous solution. This was followed by drop wise addition of $10 \mathrm{ml}$ of $\mathrm{Na}_{2} \mathrm{~S}$ under vigorous stirring for 1 hour. A chocolate brown colour precipitate was obtained which was separated by centrifugation and washed up to 6 times with double distilled water and finally with acetone. The precipitate was then dried and powdered

Volume 6 Issue 12, December 2017 


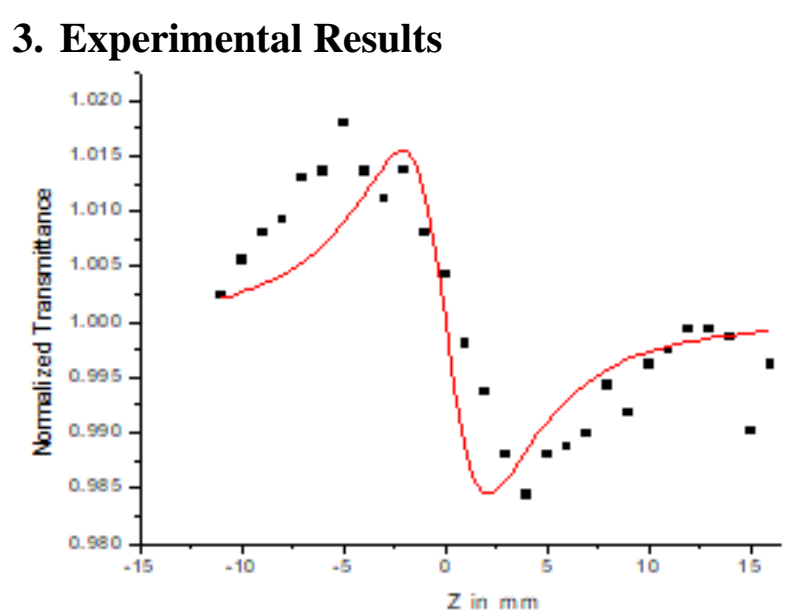

Figure 2: Closed by open aperture curve for the sample at $50 \mathrm{~mJ}$.

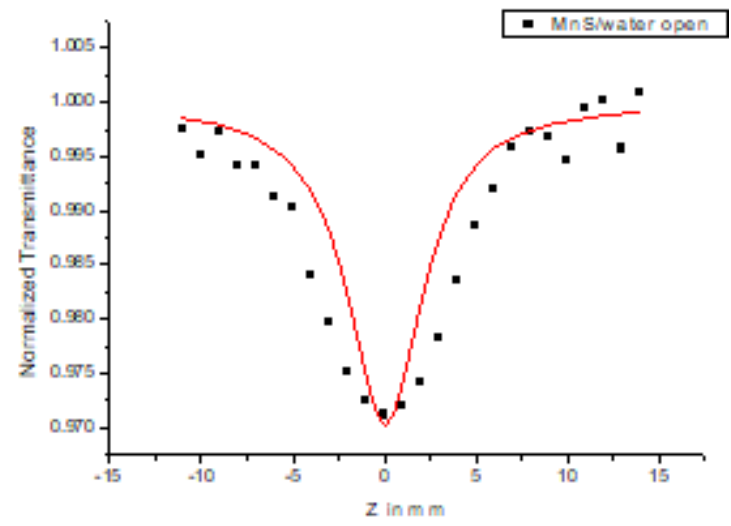

Figure 3: Open aperture curve for the sample at $50 \mathrm{~mJ}$

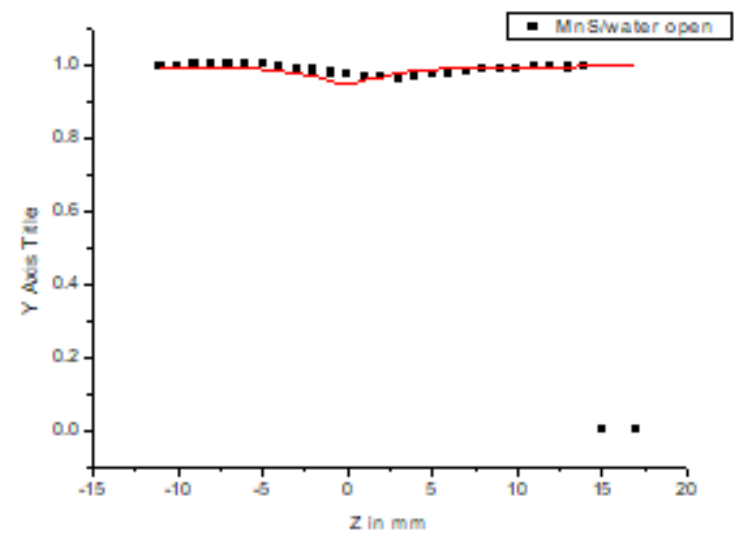

Figure 4: Closed aperture curve of the sample at $50 \mathrm{~mJ}$

The peak value configuration of the pure nonlinear curve obtained by the division method reveals a self-defocusing effect. Hence the nonlinear index of refraction will be negative. The linear and nonlinear optical parameters for the samples are listed in the table. 1

Table 1: Linear and Nonlinear optical Parameters of the

\begin{tabular}{|c|c|c|c|}
\hline Sample & $\begin{array}{c}\text { Linear } \\
\text { absorbance } \alpha \\
\text { at } 532 \mathrm{~nm}\end{array}$ & $\begin{array}{c}\text { Nonlinear } \\
\text { absorption } \\
\text { coefficient } \beta\end{array}$ & $\begin{array}{c}\text { Nonlinear } \\
\text { phase shift } \\
\Delta \emptyset\end{array}$ \\
\hline $\begin{array}{c}\text { MnS nano } \\
\text { particle in water }\end{array}$ & $0.085 \mathrm{~cm}^{-1}$ & $3.46 \mathrm{~m} / \mathrm{W}$ & 13 \\
\hline
\end{tabular}

\section{Conclusion}

The nonlinear optical parameters of the compounds were investigated by Z-scan technique using $\mathrm{Nd}$ :YAG laser having a pulse width of $7 \mathrm{~ns}$ operating at $532 \mathrm{~nm}$. The Zscan experiments on the sample were performed with and without aperture. The Z-scan curves reveals that the samples shows self defocusing effect which leads to the negative nonlinear refractive index. The result suggests that $\mathrm{MnS}$ nanoparticles are one of the suitable candidates for photonic applications.

Utilizing the NLO properties, one can design optical limiters, optical switches and guided wave structures. Another interesting area is the theoretical modeling to estimate the nonlinear properties the standardization of nonlinear optical parameters is important for all photonic applications and the modeling enables to predict the structural requirements that might be needed for this purpose.

\section{References}

[1] M. Sheik Bahae, A.A Said, and E.W. Van Stryland: High sensitivity single beam n2, measurement, Optical letters, Vol. 14, No 17, 1989, pp 955-957

[2] J.R. Goff//J.phys.B.4 (1971)116

[3] N.L Rosi and C.A. Mirkin//Chem.Rev.105 (2005)154

[4] R.W. Murray// Chem. Rev. 108(2008)2688

[5] M.C. Daniel and D.Astinic//Chem.Rev.104(2004)293 